\title{
PLAGUICIDAS ORGANOCLORADOS EN ORGANISMOS COSTEROS Y MARINOS DE LOS LITORALES MEXICANOS: UNA REVISIÓN
}

\author{
Guadalupe PONCE-VÉLEZ* y Alfonso V. BOTELLO
}

Instituto de Ciencias del Mar y Limnología, Universidad Nacional Autónoma de México

*Autora para correspondencia: ponce@cmarl.unam.mx

(Recibido febrero 2017; aceptado febrero 2018)

Palabras clave: contaminación, agroquímicos, riesgo, toxicidad, bioacumulación

\begin{abstract}
RESUMEN
Se presentan las tendencias de los plaguicidas organoclorados (POC) en organismos costeros de México, principalmente los estudiados en el Golfo de México durante aproximadamente 20 años. Los peces, crustáceos y moluscos han sido los más evaluados debido a su gran demanda para consumo humano; las familias analizadas fueron Lutjanidae, Centropomidae, Scombridae, Cichlidae, Ariidae y Carangidae para peces, Portunidae y Penaeidae para crustáceos decápodos, y Ostreidae y Mytilidae para moluscos bivalvos. El método común ha sido el propuesto por UNEP/FAO/IOC/IAEA, que tiene buena detección y confiabilidad (límite de detección $0.01 \mathrm{ng} / \mathrm{g}$ peso seco; coeficiente de variación 3-15\%; recuperación 90-101\%). Los POC más frecuentes fueron $\beta$-HCH, lindano, aldrín, $p$, $p$ '-DDT, $p$, $p$ '-DDD, heptacloro, endosulfán $\alpha, \beta$ y su sulfato. Las concentraciones han variado desde $<1.0$ hasta $\sim 20 \mathrm{ng} / \mathrm{g}$ peso seco. Para el sulfato de endosulfán, las concentraciones han sido similares para los tres grupos biológicos evaluados, sin diferencias metabólicas significativas entre invertebrados y vertebrados, lo que puede significar la gran carga de residuos clorados que existe en los ambientes costeros donde fueron capturados $\mathrm{y}$, por lo tanto, la exposición continua de la biota a estos xenobióticos. La mayoría de los POC reportados han sido prohibidos a nivel internacional y nacional; sin embargo, los datos señalan usos recientes de POC prohibidos como $p, p$ '-DDT y lindano, probablemente debido a aplicaciones clandestinas y a la compra y venta ilegal vía países donde todavía se fabrican y por rutas vulnerables de fronteras con países en desarrollo como el nuestro, representando un gran riesgo para la salud humana debido a sus características tóxicas y cancerígenas.
\end{abstract}

Key words: contamination, agrochemicals, risk, toxicity, bioaccumulation

\begin{abstract}
The trends of organochlorine pesticides (POCs) in coastal organisms of Mexico, mainly those studied in the Gulf of Mexico, are presented for approximately 20 years. Fish, crustaceans and molluscs have been the most evaluated due to their great demand for human consumption; the families analyzed were, for fish, Lutjanidae, Centropomidae, Scombridae, Cichlidae, Ariidae, Carangidae; for decapod crustaceans, Portunidae and Penaeidae, and for bivalve molluscs, Ostreidae and Mytilidae. The common method has been the one proposed by UNEP/FAO/IOC/IAEA, which has a good level of
\end{abstract}


detection and reliability (limit of detection $0.01 \mathrm{ng} / \mathrm{g}$ dry weight; coefficient of variation 3-15\%; recovery 90-101\%). The most frequent POCs were $\beta-\mathrm{HCH}$, Lindane, Aldrin, $p, p$ '-DDT, $p, p$ '-DDD, Heptachlor, Endosulfan $\alpha, \beta$ and sulphate. Concentrations range from $<1.0$ to $\sim 20 \mathrm{ng} / \mathrm{g}$ dry weight. For endosulfan sulphate, concentrations have been similar for the three biological groups evaluated, with no significant metabolic differences between invertebrates and vertebrates, which may mean that large amount of chlorinated residues exist in the coastal environments where they were captured; therefore, the continuous exposure of biota to these xenobiotics. Most reported POCs have been banned internationally and nationally, however data point to recent uses of POCs banned as $p, p$ '-DDT and Lindane, probably due to clandestine applications and illegal buying and selling via countries where manufacturing is still occurring and by vulnerable border routes with developing countries such as ours, posing a great risk to human health due to its toxic and carcinogenic characteristics.

\section{INTRODUCCIÓN}

Los plaguicidas organoclorados (POC) se han usado en México desde mediados del siglo XX en actividades agropecuarias, principalmente agrícolas, y para el control sanitario de vectores transmisores de enfermedades tropicales como el paludismo. Esto representa un panorama muy complejo para el análisis de su distribución ambiental, ya que se originan a partir de este tipo de fuentes difusas. Se ha estimado el uso de alrededor de 300 principios activos, siendo los más importantes los insecticidas, los fungicidas y los herbicidas (Rendón von Osten 2014). Los POC poseen características moleculares que favorecen su liposolubilidad y por lo tanto pueden ser bioacumulados, biomagnificados y tienen una persistencia ambiental elevada; además, se les considera como perturbadores endocrinos, ya que pueden interferir con enzimas y hormonas importantes y desencadenar procesos de carcinogénesis, ya que están contemplados como generadores potenciales de cáncer por la Agencia Internacional de Investigación sobre Cáncer de la Organización Mundial de la Salud (IARC, por sus siglas en inglés) (IARC 2011). El Catálogo Oficial de Plaguicidas en su versión actualizada 2016 contempla más de cinco mil registros de productos considerados plaguicidas y mantiene el estatus de prohibidos para los organoclorados aldrín, dieldrín y endrín, sin mencionar los de carácter restringido desde hace 25 años: lindano, DDT y endosulfán. Esto pone de manifiesto la necesidad de revisar cuidadosamente la normatividad nacional para este grupo de compuestos orgánicos persistentes de alto riesgo ambiental (DOF 1991, COFEPRIS 2016).

\section{MÉTODOS}

Los métodos utilizados por los diversos autores que han analizado muestras biológicas de organismos costeros y marinos, comparten las etapas fundamentales para la identificación y cuantificación de los POC, a saber: $a$ ) extracción con disolventes orgánicos, principalmente hexano y acetona en sistemas soxhlet, microondas, ultrasonido; concentración mediante rotaevaporación o equipo Kuderna-Danish; b) hidrólisis con $\mathrm{H}_{2} \mathrm{SO}_{4}$; c) limpieza del extracto orgánico a través de cromatografía en columna con florisil, alúmina, sílica, sulfato de sodio anhidro o con cartuchos comerciales empacados con florisil en sistema de extracción en fase sólida utilizando una elución secuencial de polaridad con hexano, cloruro de metileno, éter etílico, y por último d) análisis instrumental por cromatografía de gases generalmente con detector de captura de electrones y esporádicamente con espectrometría de masas (Holden y Marsden 1969, Canada Centre for Inland Waters 1974, UNEP 1982, 1988, 1991, UNEP/FAO/IOC/ IAEA 1986, 1991, 1993, UNEP/IOC/IAEA 1988, APHA-AWWA-WPCF 1992, Zhang et al. 2007).

\section{RESULTADOS Y DISCUSIÓN}

Se revisaron las publicaciones existentes sobre contenido de POC en diversas especies de organismos costeros y marinos de los litorales mexicanos desde hace más de 30 años, seleccionando aquellos trabajos cuyas metodologías son equiparables para fines de comparación temporal y regional en áreas que han sido sometidas a presiones ambientales 
debido a la presencia de estos agroquímicos. En los cuadros I y II se presentan los diversos trabajos encaminados a evaluar los niveles de organoclorados en diferentes grupos biológicos de organismos costeros y marinos tanto del Pacífico mexicano (PacMex) como del Golfo de México (GoM); puede observarse que la información existente en nuestro país sobre este tema tiene una antigüedad similar entre ambos litorales, ya que el grupo de Rosales y colaboradores lo inicia en Veracruz durante la década de 1970 (Rosales et al. 1979), y en Sonora y Sinaloa a principios de la década de 1980 (Rosales y Escalona 1983); a partir de entonces, se desarrollaron los estudios mayoritariamente en el sur del GoM y en el noroeste del PacMex. Se incluyen 10 trabajos científicos publicados para el Pacífico mexicano que abarcan más de 15 sitios costeros con el análisis de moluscos bivalvos como ostiones, mejillones y almejas; crustáceos como camarones y jaibas; peces como lisas, pargos y robalos, y reptiles como tortugas marinas. Para el GoM se contemplaron ocho investigaciones publicadas con datos de POC para 13 áreas costeras que evalúan los mismos grupos biológicos.

\section{Pacífico mexicano (PacMex)}

Geográficamente se ha evaluado la presencia de POC en especies costeras y marinas desde Sonora hasta Chiapas, incluyendo Baja California Sur (BCS), con excepción de la zona central de este litoral. Los ostiones considerados en los diversos trabajos pertenecen a las especies Crassostrea corteziensis, Saccostrea palmula y Crassostrea gigas. Para fines de comparación, los resultados se agruparon en la familia Ostreidae; por localidad evaluada, en la bahía de La Paz, BCS, solamente se reportaron cinco plaguicidas de la lista de 16 compuestos, siendo el isómero alfa $\mathrm{HCH}$ el que presentó la mayor concentración con $149 \mathrm{ng} / \mathrm{g}$; en orden decreciente estuvieron los isómeros beta y delta del HCH técnico con 55 y $30 \mathrm{ng} / \mathrm{g}$, respectivamente, lo que pone de manifiesto el uso de este garrapaticida comercial en el área de estudio (Cuadro III). Cabe mencionar que la mezcla comercial de este plaguicida contiene diferentes proporciones de los isómeros del hexaclorociclohexano (alfa, 50-70\%; beta, 3-14\%; gamma, 11-18\%, y delta, 6-10\%), lo que contribuye a entender la presencia dominante del primero en las muestras biológicas evaluadas. Para estos ostiones de BCS también se registraron compuestos de riesgo como endosulfán I o $\alpha$ y el metabolito $p, p$ '-DDD, este último en cantidades que sugieren un residuo antiguo del compuesto original p,p'-DDT (Osuna-López et al. 2014).
Para las especies de ostreidos de la porción continental del noroeste del Pacífico mexicano, los ecosistemas costeros de Sonora tuvieron las mayores concentraciones del $81 \%$ de los POC reportados en tejidos blandos de estos organismos, con el máximo de $3155 \mathrm{ng} / \mathrm{g}$ para el endrín aldehído, lo cual pone de manifiesto el uso de este compuesto en décadas pasadas; en segundo lugar estuvieron el dieldrín y el heptacloro, con valores de 355 y $331 \mathrm{ng} / \mathrm{g}$, respectivamente; las concentraciones decrecieron hasta llegar a $56.8 \mathrm{ng} / \mathrm{g}$ para el $p$, $p$ '-DDT. Los ostiones de los ecosistemas costeros de Sinaloa también registraron los 16 POC, aunque en magnitudes menores respecto a lo encontrado en Sonora, coincidiendo en la máxim concentración para el endrín aldehído con $141.8 \mathrm{ng} / \mathrm{g}$ (Osuna-López et al. 2014). Estos valores contrastan con el estudio realizado por Carvalho et al. (2002) en el sistema costero Ensenada del Pabellón también en Sinaloa, donde solamente reportaron el $31.5 \%$ de POC y la concentración más alta correspondió al $p, p$ '-DDE con $20 \mathrm{ng} / \mathrm{g}$, mientras que la mínima fue para el lindano con 0.7 ng/g (Cuadro III). Hacia el sur del PacMex, los ostiones de los ecosistemas costeros de Nayarit y Jalisco tuvieron en común como compuesto individual dominante al isómero delta del HCH con $1462.5 \mathrm{y}$ $639 \mathrm{ng} / \mathrm{g}$, respectivamente; en estos invertebrados nayaritas, el endosulfán II o $\beta$ fue el que presentó la mayor concentración para este grupo biológico de todo el Pacífico reportado aquí, con un valor de $319.3 \mathrm{ng} / \mathrm{g}$ sin detección confiable de su producto de degradación ambiental, el sulfato de endosulfán $(<0.01 \mathrm{ng} / \mathrm{g})$, lo cual indica un uso reciente del plaguicida comercial con categoría de restringido oficialmente (DOF 1991, Osuna-López et al. 2014).

Siguiendo con el grupo de los moluscos bivalvos, también se ha reportado presencia de POC en tejidos suaves de almejas (Megapitaria squalida, Anadara sp., Chione subrugosa) y mejillones (Mytillus strigata) para las mismas áreas de estudio consideradas en el análisis de los ostiones para este litoral mexicano, con excepción de La Paz, BCS, y las zonas costeras de Jalisco (incluyendo el ecosistema de Yavaros en Sonora) (Cuadro IV). En el inicio de la década de 1980, se encontraron pocos plaguicidas halogenados en almejas ( $M$. squalida) de Yavaros, Sonora, con valores de 7 y $7.1 \mathrm{ng} / \mathrm{g}$ como máximos para $p, p$ '-DDT y dieldrín, y concentraciones de 0.5 a $3.2 \mathrm{ng} / \mathrm{g}$ para $\Sigma \mathrm{HCH}$, epóxido de heptacloro y endrín (Rosales y Escalona 1983).

En orden creciente estuvieron las almejas ( $C h$. subrugosa) de Ensenada del Pabellón, Sinaloa, con niveles superiores a los ya comentados para Yavaros, Sonora, principalmente para sulfato de endosulfán 
CUADRO I. EVALUACIONES DEL CONTENIDO DE POC REALIZADAS EN DIVERSAS ESPECIES COSTERAS Y MARINAS DEL PACÍFICO MEXICANO

\begin{tabular}{|c|c|c|c|c|c|}
\hline Área de estudio & Estado & Grupo biológico & Especie & Nombre común & Referencia \\
\hline Punta Abreojos & $\begin{array}{l}\text { Baja California } \\
\text { Sur }\end{array}$ & Reptiles & Chelonia mydas & Tortuga verde & $\begin{array}{l}\text { Labrada-Mondra- } \\
\text { gón et al. (2011) }\end{array}$ \\
\hline Bahía Magdalena & $\begin{array}{l}\text { Baja California } \\
\text { Sur }\end{array}$ & Reptiles & Chelonia mydas & Tortuga verde & $\begin{array}{l}\text { Labrada-Mondra- } \\
\text { gón et al. (2011) }\end{array}$ \\
\hline Bahía de La Paz & $\begin{array}{l}\text { Baja California } \\
\text { Sur }\end{array}$ & Moluscos bivalvos & Saccostrea palmula & Ostión de mangle & $\begin{array}{l}\text { Osuna-López et al. } \\
\text { (2014) }\end{array}$ \\
\hline Yavaros & Sonora & Moluscos bivalvos & Megapitaria squalida & Almeja chocolata & $\begin{array}{l}\text { Rosales y Escalona } \\
\text { (1983) }\end{array}$ \\
\hline $\begin{array}{l}\text { Yavaros, } \\
\text { Moroncarit }\end{array}$ & Sonora & Crustáceos decápodos & Penaeus stylirostris & Camarón azul & $\begin{array}{l}\text { Rosales y Escalona } \\
\text { (1983) }\end{array}$ \\
\hline Yavaros & Sonora & Crustáceos decápodos & Callinectes arcuatus & Jaiba & $\begin{array}{l}\text { Rosales y Escalona } \\
\text { (1983) }\end{array}$ \\
\hline $\begin{array}{l}\text { Yavaros, } \\
\text { Moroncarit }\end{array}$ & Sonora & Peces mugílidos & $\begin{array}{l}\text { Mugil cephalus; } \\
\text { Mugil curema }\end{array}$ & Lisa, liseta & $\begin{array}{l}\text { Rosales y Escalona } \\
\text { (1983) }\end{array}$ \\
\hline $\begin{array}{l}\text { Yavaros, } \\
\text { Moroncarit }\end{array}$ & Sonora & Peces escómbridos & Scomberomorus maculatus & Sierra & $\begin{array}{l}\text { Rosales y Escalona } \\
\text { (1983) }\end{array}$ \\
\hline $\begin{array}{l}\text { Yavaros, } \\
\text { Moroncarit }\end{array}$ & Sonora & Peces planos & Paralichtys woolinaria & Lenguados & $\begin{array}{l}\text { Rosales y Escalona } \\
\text { (1983) }\end{array}$ \\
\hline $\begin{array}{l}\text { Yavaros, } \\
\text { Moroncarit }\end{array}$ & Sonora & Peces centropómidos & $\begin{array}{l}\text { Centropomus pectinatus; } \\
\text { Centropomus robalito }\end{array}$ & Robalos & $\begin{array}{l}\text { Rosales y Escalona } \\
\text { (1983) }\end{array}$ \\
\hline $\begin{array}{l}\text { Ecosistemas } \\
\text { Costeros }\end{array}$ & Sonora & Moluscos bivalvos & $\begin{array}{l}\text { Saccostrea palmula; } \\
\text { Crassostrea giga; } \\
\text { Crassostrea corteziensis }\end{array}$ & $\begin{array}{l}\text { Ostión de mangle, } \\
\text { ostión de roca }\end{array}$ & $\begin{array}{l}\text { Osuna-López et al. } \\
\text { (2014) }\end{array}$ \\
\hline $\begin{array}{l}\text { Ecosistemas } \\
\text { Costeros }\end{array}$ & Sonora & Moluscos bivalvos & $\begin{array}{l}\text { Anadara sp.; Megapitaria } \\
\text { squalida }\end{array}$ & $\begin{array}{l}\text { Almeja pata de } \\
\text { mula, almeja } \\
\text { chocolata }\end{array}$ & $\begin{array}{l}\text { Páez-Osuna et al. } \\
\text { (1998) }\end{array}$ \\
\hline $\begin{array}{l}\text { Ecosistemas } \\
\text { Costeros }\end{array}$ & Sinaloa & Moluscos bivalvos & $\begin{array}{l}\text { Saccostrea palmula; } \\
\text { Crassostrea corteziensis; } \\
\text { Sacrostrea iridescens }\end{array}$ & $\begin{array}{l}\text { Ostión de mangle, } \\
\text { ostión de roca }\end{array}$ & $\begin{array}{l}\text { Páez-Osuna et al. } \\
\text { (1998) }\end{array}$ \\
\hline $\begin{array}{l}\text { Ecosistemas } \\
\text { Costeros }\end{array}$ & Sinaloa & Moluscos bivalvos & Mytella strigata & Mejillón & $\begin{array}{l}\text { Páez-Osuna et al. } \\
\text { (1998) }\end{array}$ \\
\hline Caimanero & Sinaloa & Crustáceos decápodos & Penaeus stylirostris & Camarón azul & $\begin{array}{l}\text { Rosales y Escalona } \\
\text { (1983) }\end{array}$ \\
\hline Caimanero & Sinaloa & Crustáceos decápodos & Callinectes arcuatus & Jaiba & $\begin{array}{l}\text { Rosales y Escalona } \\
\text { (1983) }\end{array}$ \\
\hline Caimanero & Sinaloa & Peces mugílidos & $\begin{array}{l}\text { Mugil cephalus; } \\
\text { Mugil curema }\end{array}$ & Lisa, liseta & $\begin{array}{l}\text { Rosales y Escalona } \\
\text { (1983) }\end{array}$ \\
\hline Caimanero & Sinaloa & Peces centropómidos & Centropomus robalito & Robalos & $\begin{array}{l}\text { Rosales y Escalona } \\
\text { (1983) }\end{array}$ \\
\hline Bahía Ohuira & Sinaloa & Crustáceos decápodos & Penaeus vannamei & Camarón blanco & $\begin{array}{l}\text { Galindo-Reyes et } \\
\text { al. (1999) }\end{array}$ \\
\hline Bahía Ohuira & Sinaloa & Crustáceos decápodos & Penaeus vannamei & Camarón blanco & $\begin{array}{l}\text { Osuna-Flores y } \\
\text { Riva (2002) }\end{array}$ \\
\hline
\end{tabular}


CUADRO I. EVALUACIONES DEL CONTENIDO DE POC REALIZADAS EN DIVERSAS ESPECIES COSTERAS Y MARINAS DEL PACÍFICO MEXICANO

\begin{tabular}{|c|c|c|c|c|c|}
\hline Área de estudio & Estado & Grupo biológico & Especie & Nombre común & Referencia \\
\hline $\begin{array}{l}\text { Ensenada del } \\
\text { Pabellón }\end{array}$ & Sinaloa & Moluscos bivalvos & Chione subrugosa & Almeja roñosa & $\begin{array}{l}\text { Carvalho et al. } \\
\text { (2002) }\end{array}$ \\
\hline $\begin{array}{l}\text { Ensenada del } \\
\text { Pabellón }\end{array}$ & Sinaloa & Moluscos bivalvos & Mytella strigata & Mejillón & $\begin{array}{l}\text { Carvalho et al. } \\
(2002)\end{array}$ \\
\hline $\begin{array}{l}\text { Ensenada del } \\
\text { Pabellón }\end{array}$ & Sinaloa & Moluscos bivalvos & Crassostrea corteziensis & Ostión de placer & $\begin{array}{l}\text { Carvalho et al. } \\
(2002)\end{array}$ \\
\hline $\begin{array}{l}\text { Ensenada del } \\
\text { Pabellón }\end{array}$ & Sinaloa & Crustáceos decápodos & Penaeus stylirostris & Camarón azul & $\begin{array}{l}\text { Carvalho et al. } \\
\text { (2002) }\end{array}$ \\
\hline $\begin{array}{l}\text { Ensenada del } \\
\text { Pabellón }\end{array}$ & Sinaloa & Peces mugílidos & $\begin{array}{l}\text { Mugil cephalus; Mugil } \\
\text { curema }\end{array}$ & Lisa, liseta & $\begin{array}{l}\text { Carvalho et al. } \\
\text { (2002) }\end{array}$ \\
\hline $\begin{array}{l}\text { San Ignacio- } \\
\text { Navachiste- } \\
\text { Macapule }\end{array}$ & Sinaloa & Peces lutjánidos & $\begin{array}{l}\text { Lutjanus colorado; } \\
\text { Lutjanus argentiventris; } \\
\text { Lutjanus novemfasciatus }\end{array}$ & Pargos & $\begin{array}{l}\text { Granados-Galván } \\
\text { et al. (2015) }\end{array}$ \\
\hline Costa de Sinaloa & Sinaloa & Reptiles & Lepidochelys olivacea & Tortuga golfina & $\begin{array}{l}\text { García-Solorio et } \\
\text { al. (2014) }\end{array}$ \\
\hline Estuario Pozo-Rey & Nayarit & Crustáceos decápodos & Litopenaeus vannamei & Camarón blanco & $\begin{array}{l}\text { Robledo-Marenco } \\
\text { et al. }(2006)\end{array}$ \\
\hline $\begin{array}{l}\text { Estuario San } \\
\text { Cristóbal }\end{array}$ & Nayarit & Crustáceos decápodos & Litopenaeus vannamei & Camarón blanco & $\begin{array}{l}\text { Robledo-Marenco } \\
\text { et al. }(2006)\end{array}$ \\
\hline $\begin{array}{l}\text { Ecosistemas } \\
\text { Costeros }\end{array}$ & Nayarit & Moluscos bivalvos & Crassostrea corteziensis & Ostión de placer & $\begin{array}{l}\text { Osuna-López et al. } \\
\text { (2014) }\end{array}$ \\
\hline $\begin{array}{l}\text { Ecosistemas } \\
\text { Costeros }\end{array}$ & Nayarit & Moluscos bivalvos & Mytella strigata & Mejillón & $\begin{array}{l}\text { Páez-Osuna et al. } \\
\text { (1998) }\end{array}$ \\
\hline $\begin{array}{l}\text { Ecosistemas } \\
\text { Costeros }\end{array}$ & Jalisco & Moluscos bivalvos & $\begin{array}{l}\text { Crassostrea corteziensis; } \\
\text { Sacrostrea iridescens }\end{array}$ & $\begin{array}{l}\text { Ostión de placer, } \\
\text { ostión de roca }\end{array}$ & $\begin{array}{l}\text { Páez-Osuna et al. } \\
\text { (1998) }\end{array}$ \\
\hline $\begin{array}{l}\text { Laguna Chantuto- } \\
\text { Panzacola }\end{array}$ & Chiapas & Crustáceos decápodos & Penaeus vannamei & Camarón blanco & $\begin{array}{l}\text { Rueda et al. } \\
\text { (1997), Botello et } \\
\text { al. }(2000)\end{array}$ \\
\hline $\begin{array}{l}\text { Laguna Chantuto- } \\
\text { Panzacola }\end{array}$ & Chiapas & Peces lutjánidos & Lutjanus novemfasciatus & Pargos & $\begin{array}{l}\text { Rueda et al. } \\
\text { (1997), Botello et } \\
\text { al. (2000) }\end{array}$ \\
\hline $\begin{array}{l}\text { Laguna Carretas- } \\
\text { Pereyra }\end{array}$ & Chiapas & Crustáceos decápodos & Penaeus vannamei & Camarón blanco & $\begin{array}{l}\text { Rueda et al. } \\
\text { (1997), Botello et } \\
\text { al. (2000) }\end{array}$ \\
\hline
\end{tabular}

y endosulfán I, con 15 y $14 \mathrm{ng} / \mathrm{g}$, respectivamente (Carvalho et al. 2002), lo cual evidencia una mezcla en la temporalidad del uso de este agroquímico, ya que hay cantidades similares tanto del compuesto comercial para aplicación reciente como de su producto de degradación ambiental. Cabe mencionar que en estas almejas de Sinaloa se registraron tanto lindano como compuestos del grupo de los llamados "drines", todos ellos prohibidos en México desde
1991 (DOF 1991). En este orden de incremento en las concentraciones de POC reportadas para almejas, los organismos de la especie Anadara sp., evaluados en diversos ecosistemas costeros de Sonora, incluyendo Yavaros, varios años después del primer registro ya mencionado, tuvieron los niveles más altos para todos los compuestos clorados de interés en esta revisión. Hay que resaltar la magnitud del endrín aldehído con $2133 \mathrm{ng} / \mathrm{g}$, así como del sulfato de 
CUADRO II. ORGANISMOS COSTEROS Y MARINOS DE DIVERSAS LOCALIDADES DEL GOLFO DE MÉXICO EVALUADOS PARA CONOCER EL CONTENIDO DE POC EN SUS TEJIDOS.

\begin{tabular}{|c|c|c|c|c|c|}
\hline Área de estudio & Estado & Grupo biológico & Especie & Nombre común & Referencia \\
\hline Laguna Pueblo Viejo & Veracruz & Moluscos bivalvos & crassostrea virginica & Ostión americano & Rosales et al. (1979) \\
\hline $\begin{array}{l}\text { Laguna } \\
\text { Tampamachoco }\end{array}$ & Veracruz & Moluscos bivalvos & $\begin{array}{l}\text { crassostrea } \\
\text { virginica }\end{array}$ & Ostión americano & Rosales et al. (1979) \\
\hline Laguna Alvarado & Veracruz & Moluscos bivalvos & $\begin{array}{l}\text { crassostrea } \\
\text { virginica }\end{array}$ & Ostión americano & Rosales et al. (1979) \\
\hline Laguna Alvarado & Veracruz & Moluscos bivalvos & $\begin{array}{l}\text { crassostrea } \\
\text { virginica }\end{array}$ & Ostión americano & Palmerín et al. (2014) \\
\hline Laguna Alvarado & Veracruz & Moluscos bivalvos & $\begin{array}{l}\text { rangia cuneata, } \\
\text { Rangia flexuosa, } \\
\text { Polymesoda } \\
\text { caroliniana }\end{array}$ & $\begin{array}{l}\text { Almeja gallo, } \\
\text { almeja casco, } \\
\text { almeja prieta }\end{array}$ & Palmerín et al. (2014) \\
\hline Laguna El Yucateco & Tabasco & Peces cíclidos & $\begin{array}{l}\text { cichlasoma uroph- } \\
\text { thalmus, Cichla- } \\
\text { soma friedrichsthali, } \\
\text { Cichlasoma octofas- } \\
\text { ciatum, Cichlasoma } \\
\text { pearsei }\end{array}$ & $\begin{array}{l}\text { Mojarra pinta, } \\
\text { mojarra prieta, } \\
\text { mojarra castarrica }\end{array}$ & Ponce-Vélez (2012) \\
\hline Laguna El Yucateco & Tabasco & Peces megalópidos & megalops atlanticus & Sabalo & Ponce-Vélez (2012) \\
\hline Laguna El Yucateco & Tabasco & Peces centropómidos & $\begin{array}{l}\text { centropomus } \\
\text { pectinatus }\end{array}$ & Robalos & Ponce-Vélez (2012) \\
\hline Laguna El Yucateco & Tabasco & Peces mugílidos & $\begin{array}{l}\text { mugil cephalus; } \\
\text { Mugil curema }\end{array}$ & Lisa, liseta & Ponce-Vélez (2012) \\
\hline Laguna El Yucateco & Tabasco & Peces carangídos & caranx latus & Jurel & Ponce-Vélez (2012) \\
\hline Laguna El Yucateco & Tabasco & Peces gerridos & eugerres plumieri & Mojarra blanca & Ponce-Vélez (2012) \\
\hline Laguna El Yucateco & Tabasco & Peces hemúlidos & pomadasys sp. & Ronco & Ponce-Vélez (2012) \\
\hline Laguna El Yucateco & Tabasco & Peces carangídos & oligoplites saurus & Chaqueta & Ponce-Vélez (2012) \\
\hline Laguna El Yucateco & Tabasco & Peces lepisosteidos & atractosteus tropicus & Pëjelagarto & Ponce-Vélez (2012) \\
\hline Laguna El Yucateco & Tabasco & Peces aridos & ariopsis felis & Bagre & Ponce-Vélez (2012) \\
\hline Laguna El Yucateco & Tabasco & Crustáceos decápodos & $\begin{array}{l}\text { callinectes sapidus, } \\
\text { Callinectes rath- } \\
\text { bunae, Callinectes } \\
\text { similis }\end{array}$ & Jaiba azul & Ponce-Vélez (2012) \\
\hline Laguna Machona & Tabasco & Moluscos bivalvos & crassostrea virginica & Ostión americano & Rosales et al. (1979) \\
\hline Laguna Carmen & Tabasco & Moluscos bivalvos & crassostrea virginica & Ostión americano & Rosales et al. (1979) \\
\hline Estero Tamulte & Tabasco & Moluscos bivalvos & crassostrea virginica & Ostión americano & Rosales et al. (1979) \\
\hline Laguna Puerto Rico & Campeche & Moluscos bivalvos & crassostrea virginica & Ostión americano & Rosales et al. (1979) \\
\hline $\begin{array}{l}\text { Laguna de Términos } \\
\text { (Atasta) }\end{array}$ & Campeche & Moluscos bivalvos & crassostrea virginica & Ostión americano & Rosales et al. (1979) \\
\hline $\begin{array}{l}\text { Laguna de Términos } \\
\text { (Palizada) }\end{array}$ & Campeche & Moluscos bivalvos & crassostrea virginica & Ostión americano & Rosales et al. (1979) \\
\hline Estuario Río Palizada & Campeche & Crustáceos decápodos & penaeus setiferus & Camarón blanco & $\begin{array}{l}\text { Gold-Bouchot et al. } \\
\text { (1993) }\end{array}$ \\
\hline
\end{tabular}


CUADRO II. ORGANISMOS COSTEROS Y MARINOS DE DIVERSAS LOCALIDADES DEL GOLFO DE MÉXICO EVALUADOS PARA CONOCER EL CONTENIDO DE POC EN SUS TEJIDOS.

\begin{tabular}{|c|c|c|c|c|c|}
\hline Área de estudio & Estado & Grupo biológico & Especie & Nombre común & Referencia \\
\hline Estuario Río Palizada & Campeche & Moluscos bivalvos & $\begin{array}{l}\text { Crassostrea } \\
\text { virginica }\end{array}$ & Ostión americano & $\begin{array}{l}\text { Gold-Bouchot et al. } \\
\text { (1993) }\end{array}$ \\
\hline Estuario Río Palizada & Campeche & Moluscos bivalvos & $\begin{array}{l}\text { Brachidontes } \\
\text { recurvus }\end{array}$ & Mejillón curvado & $\begin{array}{l}\text { Gold-Bouchot et al. } \\
\text { (1993) }\end{array}$ \\
\hline Estuario Río Palizada & Campeche & Moluscos bivalvos & Rangia cuneata & Almeja gallo & $\begin{array}{l}\text { Gold-Bouchot et al. } \\
\text { (1993) }\end{array}$ \\
\hline Laguna de Términos & Campeche & Moluscos bivalvos & $\begin{array}{l}\text { Crassostrea } \\
\text { virginica, Crassostrea } \\
\text { rhizophora }\end{array}$ & $\begin{array}{l}\text { Ostión americano, } \\
\text { ostión de mangle }\end{array}$ & Carvalho et al. (2009) \\
\hline Laguna de Términos & Campeche & Peces lepisosteidos & Lepisosteus tropicus & Pejelagarto & Carvalho et al. (2009) \\
\hline Laguna de Términos & Campeche & Peces aridos & Ariopsis felis & Bagre & Zapata-Pérez et al. (2007) \\
\hline Costa de Campeche & Campeche & Reptiles & Chelonia mydas & Tortuga verde & $\begin{array}{l}\text { García-Besné et al. } \\
\text { (2015) }\end{array}$ \\
\hline Costa de Campeche & Campeche & Reptiles & $\begin{array}{l}\text { Eretmochelys } \\
\text { imbricata }\end{array}$ & Tortuga carey & $\begin{array}{l}\text { García-Besné et al. } \\
\text { (2015) }\end{array}$ \\
\hline Bahía de Chetumal & Qroo & Peces aridos & Ariopsis assimilis & Bagre maya & $\begin{array}{l}\text { Noreña-Barroso et al. } \\
(2004)\end{array}$ \\
\hline
\end{tabular}

CUADRO III. POC EN ESPECIES DE BIVALVOS PERTENECIENTES A LA FAMILIA OSTREIDAE (Crassostrea corteziensis, Saccostrea palmula, Crassostrea gigas Y Sacrostrea iridescens) DE DIVERSOS ECOSISTEMAS COSTEROS DEL PACÍFICO MEXICANO. VALORES EN ng/g PESO SECO.

\begin{tabular}{|c|c|c|c|c|c|c|}
\hline $\begin{array}{l}\text { Plaguicida } \\
\text { organoclorado }\end{array}$ & $\begin{array}{c}\text { 1,2 Bahía de La } \\
\text { Paz, B.C.S. }\end{array}$ & $\begin{array}{c}{ }^{1,2} \text { Ecosistemas } \\
\text { costeros de } \\
\text { Sonora }\end{array}$ & $\begin{array}{c}{ }^{1,2} \text { Ecosistemas } \\
\text { costeros de } \\
\text { Sinaloa }\end{array}$ & $\begin{array}{l}{ }^{3} \text { Ensenada del } \\
\text { Pabellón, Sin. }\end{array}$ & $\begin{array}{c}{ }^{1,2} \text { Ecosistemas } \\
\text { costeros de } \\
\text { Nayarit }\end{array}$ & $\begin{array}{c}{ }^{1,2} \text { Ecosistemas } \\
\text { costeros de } \\
\text { Jalisco }\end{array}$ \\
\hline$\alpha-\mathrm{HCH}$ & $149.0 *$ & 82.1 & 12.7 & N.R. & 51.2 & $<0.01$ \\
\hline$\beta-\mathrm{HCH}$ & 55.0 & $67.9 *$ & 18.2 & N.R. & 20.8 & 10.4 \\
\hline Lindano & $<0.01$ & $63.6^{*}$ & 5.4 & 0.7 & 5.5 & 7.8 \\
\hline$\delta-\mathrm{HCH}$ & 30.0 & 159.8 & 71.7 & N.R. & $1462.5 *$ & 639.0 \\
\hline Aldrín & $<0.01$ & $137.1 *$ & 20.4 & 0.9 & 12.1 & 17.7 \\
\hline Dieldrín & $<0.01$ & $355.0 *$ & 16.2 & N.D. & 103.7 & 10.8 \\
\hline Endrín & $<0.01$ & $125.9 *$ & 5.1 & 18.0 & $<0.01$ & 12.4 \\
\hline Endrín aldehído & $<0.01$ & 3155.0* & 141.8 & N.R. & $<0.01$ & $<0.01$ \\
\hline DDT & $<0.01$ & $56.8^{*}$ & 7.2 & 2.5 & 45.5 & 3.8 \\
\hline DDE & $<0.01$ & $216.8^{*}$ & 59.9 & 20.0 & 22.5 & 108.6 \\
\hline DDD & 24.0 & $121.7^{*}$ & 97.9 & 4.2 & 34.7 & 23.4 \\
\hline Endosulfán I & 46.9 & $179.1^{*}$ & 87.6 & N.R. & 11.2 & $<0.01$ \\
\hline Endosulfán II & $<0.01$ & 108.7 & 96.5 & N.R. & $319.3 *$ & 51.9 \\
\hline Sulfato de Endosulfán & $<0.01$ & $174.1^{*}$ & 62.4 & N.R. & $<0.01$ & $<0.01$ \\
\hline Heptacloro & $<0.01$ & $330.8 *$ & 68.4 & N.D. & 17.4 & 0.9 \\
\hline Epóxido de Heptacloro & $<0.01$ & $58.7^{*}$ & 12.5 & N.R. & $<0.01$ & 15.2 \\
\hline
\end{tabular}

${ }^{1}$ Páez-Osuna et al. (1998), ${ }^{2}$ Osuna-López et al. (2014), ${ }^{3}$ Carvalho et al. (2002), * concentración máxima por POC entre las localidades evaluadas, N.R.: no reportado por los autores, N.D.: menor al límite de detección

Los POC dominantes en la zona de estudio se destacan en letras negritas 
CUADRO IV. POC EN ESPECIES DE ALMEJAS (Megapitaria squalida, Anadara sp., Chione subrugosa) Y MEJILLONES (Mytella strigata) DE DIVERSOS ECOSISTEMAS COSTEROS DEL PACÍFICO MEXICANO. VALORES EN ng/g PESO SECO

\begin{tabular}{|c|c|c|c|c|c|c|}
\hline $\begin{array}{l}\text { Plaguicida } \\
\text { Organoclorado }\end{array}$ & $\begin{array}{l}\text { Yavaros, Son. } \\
{\text { (Almejas })^{1}}\end{array}$ & $\begin{array}{c}\text { Ecosistemas } \\
\text { costeros de } \\
\text { Sonora (Almejas) }{ }^{2}\end{array}$ & $\begin{array}{l}\text { Ensenada del } \\
\text { Pabellón, Sin. } \\
\text { (Almejas) }^{3}\end{array}$ & $\begin{array}{c}\text { Ecosistemas } \\
\text { costeros de } \\
\text { Sinaloa (Mejillones) }{ }^{2}\end{array}$ & $\begin{array}{l}\text { Ensenada del } \\
\text { Pabellón, Sin. } \\
\text { (Mejillones) }^{3}\end{array}$ & $\begin{array}{c}\text { Ecosistemas } \\
\text { costeros de } \\
\text { Nayarit (Mejillones) }\end{array}$ \\
\hline$\alpha-\mathrm{HCH}$ & N.R. & $13.5^{*}$ & N.R. & $67.8^{*}$ & N.R. & 29.4 \\
\hline$\beta-\mathrm{HCH}$ & N.R. & $34.2 *$ & N.R. & $38.3^{*}$ & N.R. & 0.02 \\
\hline Lindano & N.R. & $27.2 *$ & 1.2 & $53.7 *$ & 2.9 & 0.3 \\
\hline$\delta-\mathrm{HCH}$ & N.R. & $138.9^{*}$ & N.R. & $202.5^{*}$ & N.R. & $<0.01$ \\
\hline$\Sigma \mathrm{HCH}$ & $0.5^{*}$ & N.R. & N.R. & N.R. & N.R. & N.R. \\
\hline Aldrín & N.R. & $39.0 *$ & 2.9 & $380.0 *$ & 0.7 & $<0.01$ \\
\hline Dieldrín & 7.1 & $34.6^{*}$ & 0.3 & $43.8^{*}$ & 3.6 & $<0.01$ \\
\hline Endrín & 3.2 & $402.0 *$ & 4.3 & $<0.01$ & $14.5^{*}$ & $<0.01$ \\
\hline Endrín aldehído & N.R. & 2133.0* & N.R. & $95.6^{*}$ & N.R. & $<0.01$ \\
\hline DDT & 7.0 & $533.0 *$ & 0.3 & $4.1 *$ & 1.4 & 2.1 \\
\hline DDE & N.R. & $263.0 *$ & 1.0 & $105.8^{*}$ & 23.1 & 31.0 \\
\hline DDD & N.R. & $514.0 *$ & 1.0 & $55.1 *$ & 4.7 & 4.9 \\
\hline Endosulfán I & N.R. & $124.0 *$ & 14.0 & $109.8 *$ & N.R. & 7.4 \\
\hline $\begin{array}{l}\text { Endosulfán II } \\
\text { Sulfato de }\end{array}$ & N.R. & $92.7 *$ & 3.2 & $256.0^{*}$ & N.R. & 22.7 \\
\hline Endosulfán & N.R. & $759.0 *$ & 15.0 & $208.0 *$ & N.R. & 12.6 \\
\hline Heptacloro & N.R. & $15.3^{*}$ & N.R. & $88.6^{*}$ & 0.1 & 25.0 \\
\hline $\begin{array}{l}\text { Epóxido de } \\
\text { Heptacloro }\end{array}$ & 1.2 & $50.6^{*}$ & N.R. & $12.8^{*}$ & N.R. & $<0.01$ \\
\hline
\end{tabular}

${ }^{1}$ Rosales y Escalona (1998), ${ }^{2}$ Páez-Osuna et al. (1998), ${ }^{3}$ Carvalho et al. (2002), *concentración máxima por POC entre las localidades evaluadas, N.R.: no reportado por los autores

Los POC dominantes en la zona de estudio se destacan en letras negritas

endosulfán, $p, p^{\prime}$-DDT, $p, p$ '-DDD, endrín y lindano, con concentraciones desde 27 hasta 759 ng/g (PáezOsuna et al. 1998). En el caso de los llamados DDT, su comportamiento demuestra que probablemente el insecticida comercial sigue utilizándose para aplicaciones distintas al control de vectores de enfermedades como el paludismo, debido a que la magnitud de los valores entre el compuesto original de uso y uno de sus metabolitos es equiparable (Cuadro IV).

Los mejillones incluidos en esta revisión para el Pacífico pexicano fueron de la misma especie en los reportes considerados (M. strigata). Puede observarse que el contenido de POC en estos bivalvos evaluados en una diversidad de ambientes costeros de Sinaloa, fue mayor con relación a los de Nayarit y a un ecosistema particular de la costa sinaloense, Ensenada del Pabellón (Páez-Osuna et al. 1998), encontrándose acumulación de lindano, aldrín, dieldrín y endrín aldehído, mayoritariamente los metabolitos del DDT, el grupo del endosulfán y del heptacloro; es decir, el $100 \%$ de los compuestos de interés. Cabe resaltar que la magnitud de las concentraciones fue semejante entre los analitos ya mencionados (de 106 a 380 ng/g) (Cuadro IV). Comparativamente, los datos registrados por Carvalho et al. (2002) en esta misma especie proveniente de Ensenada del Pabellón, Sinaloa, fueron más bajos, solamente con el $50 \%$ de los compuestos detectados y un máximo de $23.1 \mathrm{ng} / \mathrm{g}$ para $p, p^{\prime}$-DDE. Las concentraciones de los mejillones pertenecientes al litoral de Nayarit fueron de magnitud similar a las ya mencionadas para Ensenada del Pabellón en Sinaloa, incluyendo la semejanza del valor más alto que fue también para el $p, p$ '-DDE con $31 \mathrm{ng} / \mathrm{g}$ y la presencia del $62.5 \%$ de este grupo de plaguicidas (Páez-Osuna et al. 1998) (Cuadro IV).

Los crustáceos se han incluido en este tipo de evaluaciones costeras debido principalmente a la importancia económica que representa el camarón para el noroeste del PacMex. En el cuadro V se presentan los datos de tres especies de este organismo bentónico (Penaeus vanname o Litopenaeus vannamei, Penaeus stylirostris y Trachypenaeus similis pacificus), así como de jaibas (Callinectes arcuatus); la mayor concentración de dieldrín se encontró en tejidos blandos de $P$. stylirostris con $1.8 \mathrm{ng} / \mathrm{g}$ y con un patrón similar al reportado para la misma especie en Caimanero, Sinaloa (Rosales y Escalona, 1983). En contraste con los primeros reportes de POC en camarones de Sinaloa, están los datos de Galindo et al. (1999) y 


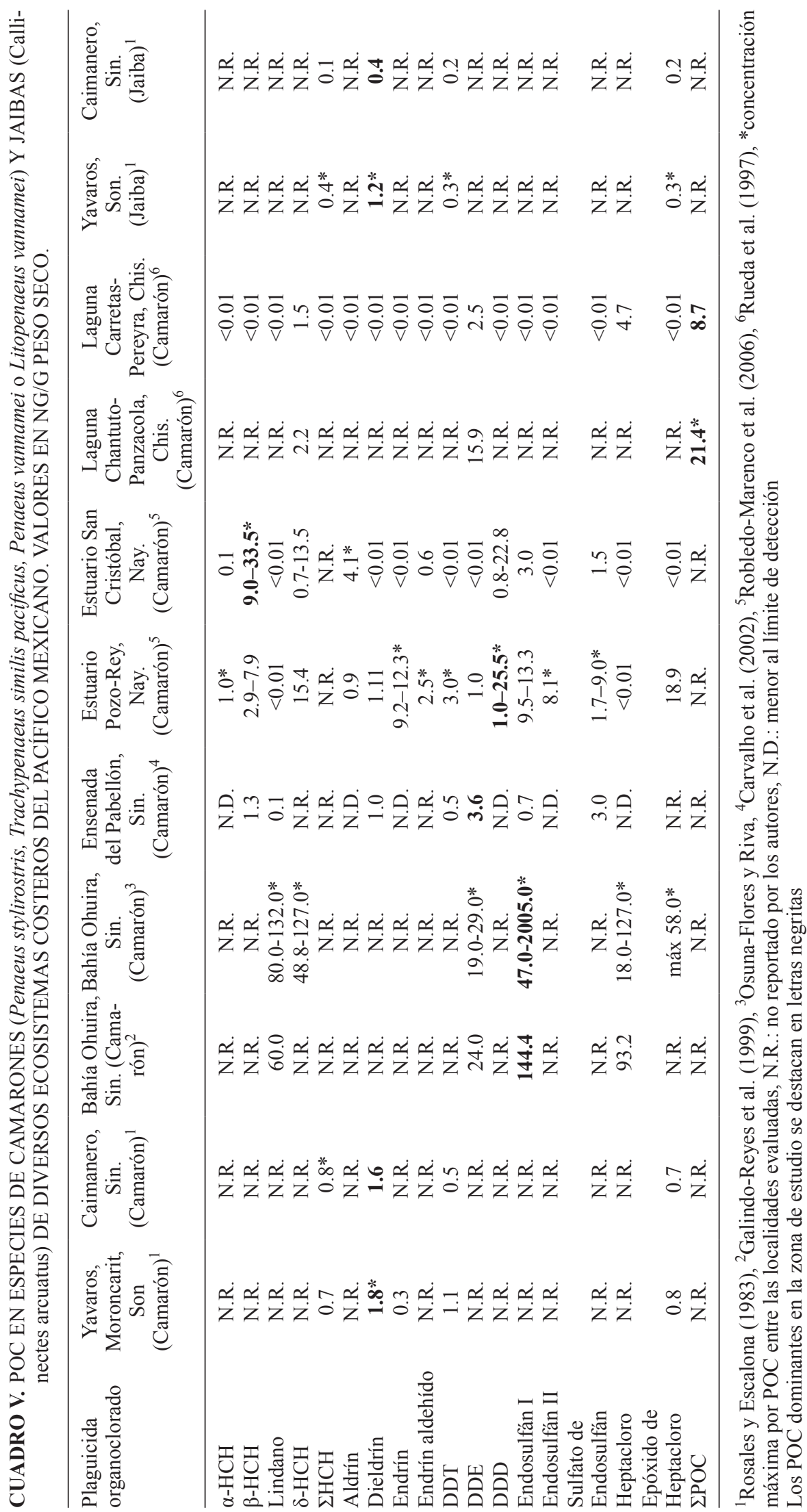


Osuna-Flores y Riva (2002) para P. vannamei de la Bahía de Ohuira, que muestran un contraste importante en la magnitud de las concentraciones obtenidas por estos grupos de investigación. Los mencionados en primer lugar obtuvieron niveles superiores a los de Rosales y Escalona (1983) principalmente para lindano, $p, p$ '-DDE, endosulfán I y heptacloro con 60, 24, 144.4 y $93.2 \mathrm{ng} / \mathrm{g}$, respectivamente; comparativamente, el trabajo de 2002 reportó concentraciones todavía mayores, con máximos de 132, 127, 29, 2005, 127 y 58 ng/g para lindano, delta-HCH, $p, p$ '-DDE, endosulfán I, heptacloro y epóxido de heptacloro, respectivamente. Carvalho et al. (2002) registraron concentraciones menores de lindano, dieldrín, $p, p$ '-DDE y endosulfán I para $P$. stylirostris en Ensenada del Pabellón, con $0.1,1.0,3.6$ y $0.7 \mathrm{ng} / \mathrm{g}$, respectivamente (Cuadro V). Hacia el sur del PacMex, Robledo-Marenco et al. (2006) evaluaron el contenido de organoclorados en camarones de dos ecosistemas costeros de Nayarit; estos autores reportan concentraciones intermedias en relación con los trabajos anteriormente descritos. Los organismos del estuario Pozo-Rey presentaron mayores niveles de delta-HCH, dieldrín, endrín, endrín aldehído, DDT, endosulfán I y II, y sulfato de endosulfán, respecto a la misma especie obtenida en el estuario San Cristóbal, ambos en la región costera de San Blas, Nayarit, en tanto que en este último sitio la concentración más alta fue para beta-HCH y aldrín. Es importante resaltar el hecho de que se presentó una mayor cantidad de POC en los camarones de los ecosistemas nayaritas respecto a los organismos de las localidades restantes incluidas en esta compilación (Cuadro V). Finalmente, las jaibas $C$. arcuatus provenientes de Yavaros, Sonora, y Caimanero, Sinaloa, tuvieron un bajo contenido de plaguicidas organoclorados y la magnitud de aquellos que se encontraron fue similar, resaltando la presencia de dieldrín, $p, p$ '- $D D T$ y epóxido de heptacloro con concentraciones de 0.2 a 1.2 $\mathrm{ng} / \mathrm{g}$. Hay que mencionar el bajo contenido de POC en los camarones peneidos de Chiapas, ya que solamente se detectaron concentraciones del isómero delta-HCH y el metabolito aromático $p, p$ '-DDE, siendo mayores los niveles en el sistema lagunar Chantuto-Panzacola que en el complejo costero de Carretas-Pereyra, ambos ecosistemas considerados grandes productores de camarones en esta región del Pacífico sur de México (Rueda et al. 1997, Botello et al. 2000).

Dentro del grupo de los vertebrados costeros y marinos, se han incluido principalmente peces y algunos reptiles como tortugas marinas para estudiar el contenido de estos agroquímicos en diversos tejidos, ya que los primeros tienen una gran importancia económica como recurso pesquero y alimenticio, y los se- gundos pertenecen al grupo de las especies en peligro de extinción. En el cuadro VI se puede observar que, para los primeros registros de peces reportados por Rosales y Escalona (1983) en el litoral de Sonora (Yavaros y Moroncarit) y Sinaloa (Caimanero) se presentaron concentraciones de compuestos clorados similares en magnitud y diversidad, dominando en Yavaros el dieldrín en las lisas Mugil cephalus y $M$. curema, con un promedio de $5 \mathrm{ng} / \mathrm{g}$, mientras que en Caimanero el nivel más alto en $M$. cephalus fue para el p,p'-DDT, con $6.6 \mathrm{ng} / \mathrm{g}$. En 2002, el trabajo de Carvalho et al. (2002) realizado en Ensenada del Pabellón, Sinaloa, incluyó el análisis de las branquias de peces de la misma especie de lisa (M. cephalus). Estos investigadores encontraron concentraciones superiores en este órgano blanco, con un máximo de $530 \mathrm{ng} / \mathrm{g}$ para el sulfato de endosulfán y mayor variedad de compuestos xenobióticos como el $p, p$ '-DDE, endosulfán II, $p, p$ '-DDT y beta-HCH, con 450, 52, 38 y $34 \mathrm{ng} / \mathrm{g}$, respectivamente. En contraste, estos mismos autores reportaron valores menores de POC en el tejido muscular de los mismos organismos analizados; por ejemplo, el máximo fue de $16 \mathrm{ng} / \mathrm{g}$ para $p, p$ '-DDE. El resto de los compuestos detectados tuvo concentraciones $<3.5 \mathrm{ng} / \mathrm{g}$ y la diversidad de plaguicidas también fue más baja, patrón que concuerda con lo reportado para otros estudios, lo cual demuestra una acumulación diferencial en los organismos (Boonyatumanond et al. 2002, Cakirogullari y Secer 2011).

Recientemente, Granados-Galván et al. (2015) analizaron diferentes especies de pargos (género Lutjanus) provenientes del sistema costero San IgnacioNavachiste-Macapule en Sinaloa, y encontraron una presencia importante de POC en los tejidos de estos peces, similar a la reportada para las lisas de Ensenada del Pabellón del mismo estado mexicano, con diferencias en la magnitud de las concentraciones, ya que en este trabajo reciente la máxima encontrada fue para el isómero delta del $\mathrm{HCH}$ con $31.3 \mathrm{ng} / \mathrm{g}$, con presencia de los tres integrantes de la familia aromática, siendo el de mayor grado el $p, p$ '-DDT (7 ng/g); también se registraron aldrín, lindano y los grupos del endosulfán y del heptacloro, lo cual denota aplicaciones recientes de este tipo de compuestos prohibidos (Cuadro VI). Hacia el sur del PacMex, Rueda et al. (1997) evaluaron el contenido de estos POC en tejido muscular del pargo Lutjanus novemfasciatus en Chiapas y reportaron solamente cinco compuestos detectados, en comparación con los 13 plaguicidas reportados por Granados-Galván et al. (2015), siendo el aldrín el de mayor nivel con $39.4 \mathrm{ng} / \mathrm{g}$, seguido por el epóxido de heptacloro, el endosulfán I y el $p, p$ '-DDT con $23.3,13.1 \mathrm{y}$ 
CUADRO VI. POC EN PECES (Mugil cephalus, Mugil curema, Scomberomorus maculatus, Paralichtys woolinaria, Centropomus pectinatus, Centropomus robalito, Lutjanus corolado, Lutjanus argentiventris Y Lutjanus novemfasciatus) Y TORTUGAS MARINAS (Lepidochelys olivacea Y Chelonia mydas) DE DIVERSOS ECOSISTEMAS COSTEROS DEL PACÍFICO MEXICANO. VALORES EN ng/g PESO SECO.

\begin{tabular}{|c|c|c|c|c|c|c|c|c|c|}
\hline $\begin{array}{l}\text { Plaguicida } \\
\text { organoclorado }\end{array}$ & $\begin{array}{l}\text { Yavaros, } \\
\text { Moroncarit, } \\
\text { Son. }\left(\text { Pez) }{ }^{1}\right.\end{array}$ & $\begin{array}{l}\text { Caimanero, } \\
\text { Sin. }(\text { Pez })^{1}\end{array}$ & $\begin{array}{c}\text { Ensenada } \\
\text { del } \\
\text { Pabellón, } \\
\text { Sin. (Pez, } \\
\text { branquias) }\end{array}$ & $\begin{array}{l}\text { Ensenada } \\
\text { del } \\
\text { Pabellón, } \\
\text { Sin. (Pez) }\end{array}$ & $\begin{array}{l}\text { San Ignacio- } \\
\text { Navachiste- } \\
\text { Macapule, } \\
\text { Sin. (pez) }\end{array}$ & $\begin{array}{c}\text { Laguna } \\
\text { Chantuto- } \\
\text { Panzacola, } \\
\text { Chis. (Pez) }\end{array}$ & $\begin{array}{l}\text { Costa de } \\
\text { Sinaloa } \\
\text { (Tortuga } \\
\text { Golfina, } \\
\text { Huevos) }\end{array}$ & $\begin{array}{l}\text { Punta Abre- } \\
\text { ojos, B.C.S. } \\
\text { (Tortuga } \\
\text { Verde, } \\
\text { Plasma) }\end{array}$ & $\begin{array}{c}\text { Bahía } \\
\text { Magdalena, } \\
\text { B.C.S. } \\
\text { (Tortuga } \\
\text { Verde, } \\
\text { Plasma) }\end{array}$ \\
\hline$\alpha-\mathrm{HCH}$ & N.R. & N.R. & $5.1 *$ & 0.1 & 2.0 & N.R. & N.R. & 0.2 & $1.3^{*}$ \\
\hline$\beta-\mathrm{HCH}$ & N.R. & N.R. & $34.0^{*}$ & 3.2 & 3.0 & N.R. & $980.0^{*}$ & 1.2 & 4.6 \\
\hline Lindano & N.R. & N.R. & $5.4^{*}$ & 0.2 & 2.0 & N.R. & $1530.0 *$ & 0.8 & 3.0 \\
\hline$\delta-\mathrm{HCH}$ & N.R. & N.R. & N.R. & N.R. & $31.3 *$ & N.R. & N.R. & 0.8 & $2.6^{*}$ \\
\hline$\Sigma \mathrm{HCH}$ & $1.2 *$ & 1.1 & N.R. & N.R. & N.R. & N.R. & N.R. & N.R. & N.R. \\
\hline Aldrín & 0.4 & N.R. & 2.8 & N.D. & 5.8 & $39.4 *$ & N.R. & 1 & $10.4 *$ \\
\hline Dieldrín & 5.0 & 1.2 & $13.0^{*}$ & 1.3 & N.R. & N.R. & N.R. & $<0.001$ & $<0.001$ \\
\hline Endrín & 4.4 & N.R. & $19.0 *$ & 0.1 & N.R. & N.R. & $120.0^{*}$ & 1.1 & $<0.001$ \\
\hline Endrín aldehído & N.R. & N.R. & N.R. & N.R. & N.R. & N.R. & $120.0^{*}$ & $<0.001$ & $<0.001$ \\
\hline DDT & 2.4 & 6.6 & $38.0^{*}$ & 2.2 & 7.0 & 12.3 & N.R. & $0.001-0.3$ & $0.001-4.0^{*}$ \\
\hline DDE & N.R. & N.R. & $450.0^{*}$ & 16.0 & 3.3 & N.R. & $30.0^{*}$ & $<0.001$ & $<0.001$ \\
\hline DDD & N.R. & N.R. & $16.0^{*}$ & N.D. & 2.7 & N.R. & 10.0 & $<0.001$ & $0.005-11.8^{*}$ \\
\hline Endosulfán I & N.R. & N.R. & $16.0^{*}$ & 1.3 & 2.1 & N.R. & $170.0^{*}$ & $<0.001$ & $0.005-1.2$ \\
\hline $\begin{array}{l}\text { Endosulfán II } \\
\text { Sulfato de }\end{array}$ & N.R. & N.R. & $52.0 *$ & 0.9 & 6.0 & 13.1 & N.R. & $<0.001$ & $0.005-7.5^{*}$ \\
\hline Endosulfán & N.R. & N.R. & $530.0 *$ & 2.4 & 7.7 & N.R. & N.R. & $<0.001$ & $0.005-13.0^{*}$ \\
\hline Heptacloro & N.R. & N.R. & 0.4 & N.D. & 4.4 & $5.9^{*}$ & $30.0^{*}$ & 8.6 & 2.7 \\
\hline $\begin{array}{l}\text { Epóxido de } \\
\text { Heptacloro }\end{array}$ & 1.3 & 0.4 & N.R. & N.R. & 6.0 & $23.3^{*}$ & N.R. & $0.005-7.9^{*}$ & $0.005-1.7$ \\
\hline$\Sigma \mathrm{POC}$ & N.R. & N.R. & N.R. & N.R. & 19.3 & $93.9 *$ & N.R. & N.R. & N.R. \\
\hline
\end{tabular}

${ }^{1}$ Rosales y Escalona (1983), ${ }^{2}$ Carvalho et al. (2002), ${ }^{3}$ Granados-Galván et al. (2015), ${ }^{4}$ Rueda et al. (1997), ${ }^{5}$ García-Solorio et al. (2004), ${ }^{6}$ Labrada-Mondragón et al. (2011), *concentración máxima por POC entre las localidades evaluadas, N.R.: no reportado por los autores, N.D.: menor al límite de detección

Los POC dominantes en la zona de estudio se destacan en letras negritas

$12.3 \mathrm{ng} / \mathrm{g}$, respectivamente. Esto evidencia nuevamente el uso ilegal de productos prohibidos desde el inicio de la década de 1990 (Botello et al. 2000).

Respecto a las tortugas marinas consideradas para evaluar la presencia de diversos POC, García-Solorio et al. (2014) analizaron huevos de tortuga golfina (Lepidochelys olivacea) en la costa de Sinaloa, encontrando concentraciones muy altas para lindano y para el isómero beta de esa mezcla técnica, con un máximo de $1530 \mathrm{ng} / \mathrm{g}$ y $980 \mathrm{ng} / \mathrm{g}$, respectivamente. Esto significa un uso continuo y reciente de estos alicíclicos en México, ya que su transferencia hacia la descendencia de dicha tortuga implica la adquisición de estos hidrocarburos clorados a través del alimento (pastos marinos, crustáceos, moluscos, peces pequeños), lo cual demuestra un proceso de biomagnificación. La presencia del endrín y su forma aldehído en la misma concentración, al igual que endosulfán I y heptacloro, confirman la aplicación en fechas recientes de estos compuestos. De forma contrastante, Labrada-Martagón et al. (2011) registraron concentraciones muy bajas en plasma de tortuga verde (Chelonia mydas) proveniente de BCS, con valores de 0.005 a $8.6 \mathrm{ng} / \mathrm{g}$ en los organismos de Punta Abreojos y de 0.001 a 10.4 ng/g en Bahía Magdalena, correspondiendo a heptacloro y aldrín las mayores concentraciones en cada uno de los sitios costeros mencionados. Estos trabajos ponen en perspectiva la alta liposolubilidad de los POC (huevos vs. plasma) así como su acumulación magnificada debido a la posición trófica de las tortugas marinas y a la composición mixta de su dieta, ya que se comportan como especies carnívoras en los primero años de vida en mar abierto y migran a hábitos herbívoros cuando regresan a la costa para reproducirse y desovar (Gardner et al. 2003) (Cuadro VI). 


\section{Golfo de México (GoM)}

Comparativamente con el PacMex, en el GoM se cuenta con menos localidades estudiadas para registrar la presencia de plaguicidas organoclorados mediante el análisis de diversos organismos costeros y marinos. En el cuadro VII se presenta la compilación de concentraciones de POC en moluscos bivalvos de importancia comercial y de alto consumo en este litoral. Para los ostiones de la especie Crassostrea virginica, recurso económico muy importante en el GoM, se cuenta con registros tan antiguos como los reportados por Rosales et al. (1979) para ostiones de diversas lagunas costeras, desde Veracruz hasta Campeche. Puede observarse que este primer registro sobre organoclorados se enfocó solamente a tres compuestos, dieldrín, endrín y $p, p$ '-DDT, debido a la instrumentación analítica que en esa época permitía la identificación confiable de un número limitado de analitos halogenados. En este trabajo pionero, el $p, p$ '-DDT fue el compuesto que tuvo concentraciones mayores, con el máximo en los bivalvos obtenidos en el estero Tamulte de Tabasco con $28 \mathrm{ng} / \mathrm{g}$, siguiéndole los ostiones de la laguna Carmen del mismo estado y los de Pueblo Viejo al norte de Veracruz con 17 y $16 \mathrm{ng} / \mathrm{g}$, respectivamente. Este registro histórico pone de manifiesto el uso indiscriminado de este insecticida en diversas zonas costeras mexicanas para el control del mosco a nivel doméstico, turístico y sanitario. Hay que resaltar la presencia de dieldrín ya desde esta época, sin existir la normatividad sobre la prohibición de este xenobiótico. El alto contenido en tejido blando de estos ostiones se registró para los organismos de la laguna Puerto Rico en Campeche, sistema aledaño a la laguna de Términos, con 3.3 $\mathrm{ng} / \mathrm{g}$, seguido por el detectado para los bivalvos de la laguna de Tampamachoco en el norte de Veracruz, con $1.1 \mathrm{ng} / \mathrm{g}$. Aproximadamente 15 años más tarde, Gold-Bouchot et al. (1993) estudiaron ejemplares de C. virginica provenientes del río Palizada y de la laguna de Términos en Campeche; sus resultados mostraron una mayor diversidad de compuestos en los ostiones obtenidos del sistema lagunar y concentraciones más altas en los organismos muestreados en el río, ya que, por ejemplo, el endrín tuvo un valor de 56.7 en el Palizada contra $0.4 \mathrm{ng} / \mathrm{g}$ en Términos, patrón semejante para lindano, aldrín y $p, p$ '-DDD. Sin embargo, cabe resaltar que en los ostiones lagunares se registraron los grupos del endosulfán y del DDT completos, así como heptacloro y dieldrín, compuestos que no fueron reportados para los bivalvos de Palizada (Cuadro VII).

Palmerín et al. (2014) evaluaron la presencia de estos agroquímicos en el sistema lagunar de Alvarado,
Veracruz, en las diversas matrices ambientales, incluyeron bivalvos del grupo de los ostiones $(C$. virginica) y de las almejas (Rangia cuneata, Rangia flexuosa, Polymesoda caroliniana). Los resultados de esta investigación reciente contrastan con la original de 1979: en la actual se reportó una presencia significativa de POC (94\%), así como concentraciones mayores de las registradas inicialmente; el $p, p$ '-DDT se duplicó, mientras que el dieldrín y el endrín se incrementaron 15 y 30 veces, respectivamente, respecto al valor histórico, lo cual pone en evidencia el avance tecnológico para detectar niveles cada vez menores $\mathrm{y}$, lo más importante, el uso continuo de estos compuestos persistentes de gran riesgo ambiental como lo demuestran la presencia de lindano y del grupo de los “drines" prohibidos en México desde 1991 (DOF 1991). Otro aspecto relevante, resultado de contar con mayor capacidad de detección e identificación instrumental, es el hecho de obtener información de los productos de transformación de algunos POC, como es el caso de la familia aromática o del DDT; precisamente con estos datos, se tiene registro de que en Alvarado, Veracruz, el contenido mayoritario de $p, p$ '-DDT respecto a DDD y DDE pone de manifiesto un uso reciente de este insecticida, lo cual cuestiona el compromiso de nuestro país para erradicarlo del territorio nacional (SEMARNAT-ONUDI-IPN 2016).

Las almejas incluidas en el cuadro VII, fueron estudiadas en el río Palizada, Campeche (Gold-Bouchot et al. 1993) y en Alvarado, Veracruz (Palmerín et al. 2014) con aproximadamente 20 años de diferencia. Se observa un fenómeno semejante al ya descrito para C. virginica: hubo mayor diversidad de compuestos en el registro reciente que en el de principios de la década de 1990 ya que en el pionero se presentaron solamente dos plaguicidas, lindano y $p$, $p$ '-DDD, este último con mayor concentración $(16.8 \mathrm{ng} / \mathrm{g})$, mientras que en las almejas de Veracruz se detectaron los 16 POC de interés ambiental, con dominio del endrín aldehído (13.6 ng/g), sin dejar de lado la presencia de los "drines", además de tener evidencia de un uso reciente de DDT así como de un componente residual de este insecticida ( $p, p$ '-DDT $11 \mathrm{ng} / \mathrm{g} ; p, p^{\prime}$-DDE $11.7 \mathrm{ng} / \mathrm{g}$; $p$, $p$ '-DDD $3.6 \mathrm{ng} / \mathrm{g}$ ). Ambos ecosistemas costeros, Palizada y Alvarado, han estado sometidos a presiones pesqueras y de eventos de paludismo, por lo que el control de vectores ha ocurrido desde la década de 1970. El uso de este plaguicida ha sido probablemente más intenso en el sur de Veracruz, ya que es una región con mayor actividad agrícola (enfocada principalmente al cultivo de la caña de azúcar) y también con un giro ganadero de mayor envergadura que la zona aledaña al Palizada, lo cual 


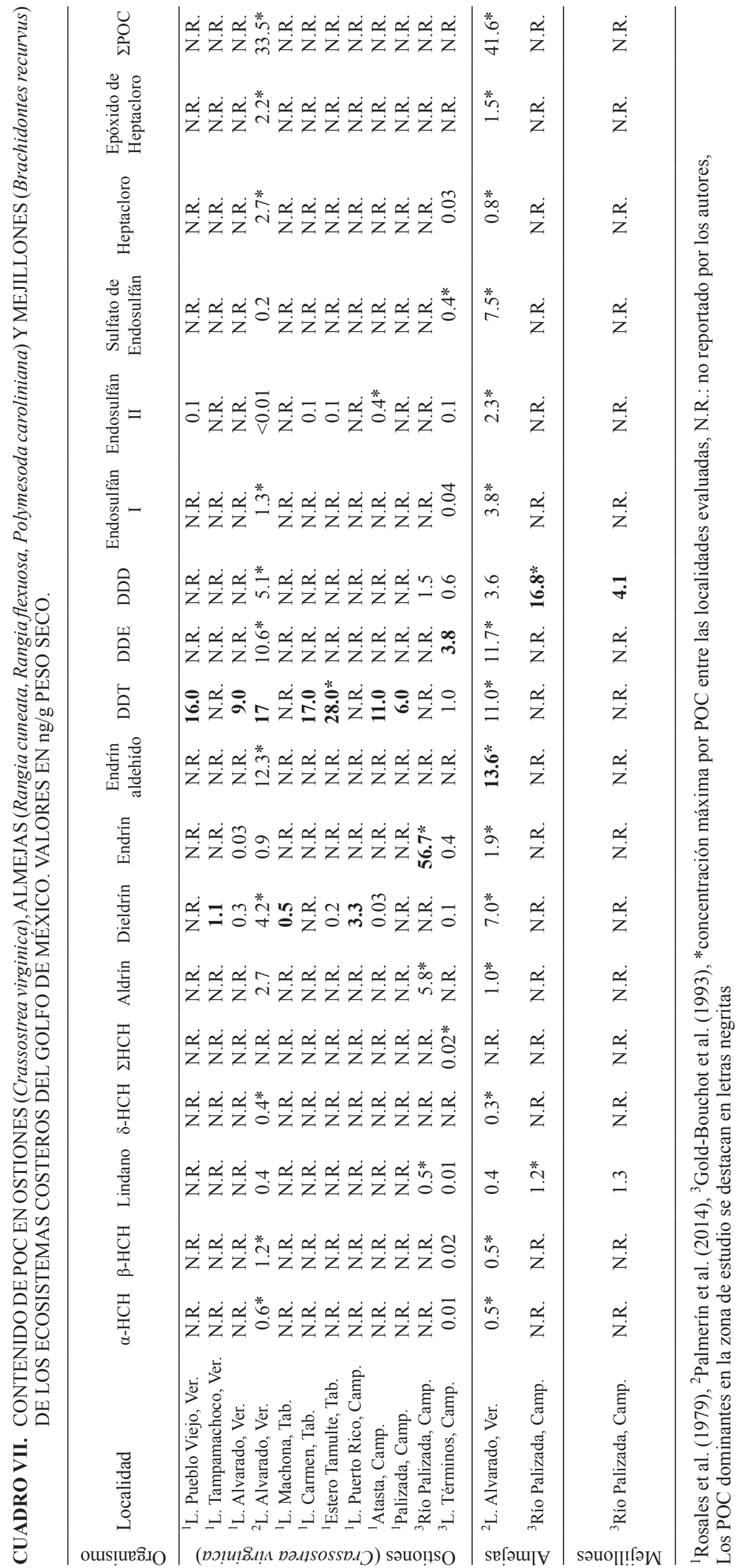


contribuye sin lugar a dudas a una carga importante de agroquímicos hacia los ecosistemas costeros.

Los mejillones (Brachidontes recurvus) incluidos en el cuadro VII fueron estudiados por Gold-Bouchot et al. (1993) y al igual que para las almejas reportadas por estos autores, solamente se encontraron dos compuestos, lindano y $p, p^{\prime}$-DDD, siendo este último el de mayor concentración con $4.1 \mathrm{ng} / \mathrm{g}$, superior a lo reportado para C. virginica e inferior a lo registrado para las almejas. La magnitud de la concentración de lindano fue equiparable tanto para los mejillones como para las almejas y ambas especies superaron a los ostiones estudiados por el mismo grupo de trabajo.

Los crustáceos también han sido objeto de estudio con relación al contenido de POC en sus tejidos suaves y en sus cubiertas o exoesqueletos. En el cuadro VIII se presentan los datos compilados desde 1993 en conjunto con los reportados para peces y tortugas marinas hasta 2015. Respecto al grupo de los decápodos, se cuenta con el trabajo de Gold-Bouchot et al. (1993) para el camarón blanco Penaeus setiferus del río Palizada, Campeche, donde no se detectaron plaguicidas organoclorados. En contraste, PonceVélez (2012) encontró en tres especies de jaibas (Callinectes sapidus, C. similis y C. rathbunae) de la laguna El Yucateco, Tabasco, 81 \% de los POC de riesgo ambiental, en concentraciones bajas de 0.03 a $2.7 \mathrm{ng} / \mathrm{g}$; este máximo correspondió al sulfato de endosulfán, resaltando el hecho de que se detectaron dieldrín y $p, p$ '-DDT.

Los peces se han incluido por son un recurso económico y alimenticio importante para la región costera del GoM, y en algunos sitios de gran vulnerabilidad social representan el insumo nutricional básico. Ponce-Vélez (2012) evaluó la presencia de diversos contaminantes orgánicos persistentes, entre ellos los POC, en una gama muy amplia de peces que incluyó mojarras (Cíclidos), sábalos (Megalópidos), lisas (Mugílidos), jureles (Carángidos), robalos (Centropómidos), bagres (Áridos) y pejelagartos (Lepisosteidos), entre otros, encontrando el

CUADRO VIII. POC EN JAIBAS (Callinectes sapidus, C. similis Y C. rathbunae), CAMARONES (Penaeus setiferus), PECES (Cíclidos, Mugílidos, Centropómidos, Carángidos, Aridos, Lepisosteidos) Y TORTUGAS MARINAS (Chelonia mydas, Eretmochelys imbricata) DE LA ZONA COSTERA DEL GOLFO DE MÉXICO. VALORES EN ng/g PESO SECO

\begin{tabular}{|c|c|c|c|c|c|c|c|c|c|c|}
\hline $\begin{array}{l}\text { Plaguicida } \\
\text { organoclorado }\end{array}$ & $\begin{array}{c}\text { L. El } \\
\text { Yucateco, } \\
\text { Tab. } \\
\text { (Jaiba) }^{1}\end{array}$ & $\begin{array}{c}\text { Río } \\
\text { Palizada, } \\
\text { Camp. } \\
\text { (Camarón) }{ }^{2}\end{array}$ & $\begin{array}{c}\text { L. El } \\
\text { Yucateco, } \\
\text { Tab. } \\
\text { (Peces) })^{1}\end{array}$ & $\begin{array}{c}\text { L. } \\
\text { Términos, } \\
\text { Camp. } \\
\text { (Peces) })^{3}\end{array}$ & $\begin{array}{c}\text { L. } \\
\text { Términos, } \\
\text { Camp. } \\
\text { (Pejelagarto) }\end{array}$ & $\begin{array}{c}\text { Bahía de } \\
\text { Chetumal, } \\
\text { Qroo } \\
(\text { Peces })^{5}\end{array}$ & $\begin{array}{c}\text { Costa de } \\
\text { Campeche } \\
\text { (Tortuga } \\
\text { Verde } \\
\text { sangre) }\end{array}$ & $\begin{array}{c}\text { Costa de } \\
\text { Campeche } \\
\text { (Tortuga } \\
\text { verde } \\
\text { huevos) }^{6}\end{array}$ & $\begin{array}{l}\text { Costa de } \\
\text { Campeche } \\
\text { (Tortuga } \\
\text { carey } \\
\text { sangre) }\end{array}$ & $\begin{array}{c}\text { Costa de } \\
\text { Campeche } \\
\text { (Tortuga } \\
\text { carey } \\
\text { huevos) }\end{array}$ \\
\hline$\alpha-\mathrm{HCH}$ & $<0.01$ & N.D. & 0.4 & N.R. & N.R. & $43.7 *$ & N.R. & 25.6 & 1.9 & 140.0 \\
\hline$\beta-\mathrm{HCH}$ & 0.2 & N.D. & 0.2 & N.R. & N.R. & $27.0 *$ & N.R. & 164.9 & N.R. & N.R. \\
\hline Lindano & 0.03 & N.D. & 0.1 & 6.9 & N.R. & $46.4 *$ & 4.4 & N.R. & 2.9 & 229.4 \\
\hline$\delta-\mathrm{HCH}$ & 0.4 & N.D. & $2.0^{*}$ & N.R. & N.R. & N.R. & N.R. & 144.7 & 1.9 & 296.5 \\
\hline$\Sigma \mathrm{HCH}$ & N.R. & N.D. & N.R. & 30.0* & N.R. & N.R. & N.R. & N.R. & N.R. & N.R. \\
\hline Aldrín & $<0.01$ & N.D. & 1.5 & N.R. & N.R. & $277.6^{*}$ & 0.9 & N.R. & 2.3 & 757.9 \\
\hline Dieldrín & 0.5 & N.D. & 0.6 & N.R. & 0.1 & $32.9^{*}$ & N.R. & N.R. & N.R. & 344.3 \\
\hline Endrín & $<0.01$ & N.D. & 0.5 & N.R. & N.R. & $40.0 *$ & 7.9 & N.R. & 5.2 & 1296.0 \\
\hline Endrín aldehído & 0.5 & N.D. & $0.4^{*}$ & N.R. & N.R. & N.R. & N.R. & 220.8 & N.R. & 564.6 \\
\hline DDT & 0.7 & N.D. & 1.9 & N.R. & 3.0 & $84.9 *$ & 4.3 & 487.3 & 3.9 & 898.4 \\
\hline DDE & 0.1 & N.D. & 0.7 & N.R. & 8.5 & $315.3^{*}$ & 3.4 & 38.7 & 3.6 & 410.5 \\
\hline DDD & 0.2 & N.D. & 0.3 & N.R. & 2.3 & 428.0* & 4.7 & 89.7 & 15.4 & 2398.0 \\
\hline Endosulfán I & 0.3 & N.D. & $0.7^{*}$ & N.R. & N.R. & N.R. & N.R. & 493.2 & N.R. & N.R. \\
\hline $\begin{array}{l}\text { Endosulfán II } \\
\text { Sulfato de }\end{array}$ & 0.3 & N.D. & 0.6 & N.R. & N.R. & $26.6^{*}$ & 6.8 & 179.2 & 4.4 & N.R. \\
\hline Endosulfán & 2.7 & N.D. & $5.4^{*}$ & N.R. & 0.03 & N.R. & 5.7 & N.R. & 4.6 & N.R. \\
\hline Heptacloro & 0.04 & N.D. & 1.2 & N.R. & N.R. & $48.0 *$ & N.R. & N.R. & N.R. & 173.5 \\
\hline $\begin{array}{l}\text { Epóxido de } \\
\text { Heptacloro }\end{array}$ & 0.3 & N.D. & 0.5 & N.R. & N.R. & $31.6^{*}$ & 2.1 & 78.3 & 19.4 & 1111.0 \\
\hline$\Sigma \mathrm{POC}$ & 6.5 & N.D. & 9.8 & N.R. & N.R. & $795.8^{*}$ & N.R. & N.R. & N.R. & N.R. \\
\hline
\end{tabular}

${ }^{1}$ Ponce-Vélez (2012), ${ }^{2}$ Gold-Bouchot et al. (1993), ${ }^{3}$ Zapata-Pérez et al. (2007), ${ }^{4}$ Carvalho et al. (2009), ${ }^{5}$ Noreña-Barroso et al. (2004), ${ }^{6}$ García-Besné et al. (2015), *concentración máxima por POC entre las localidades evaluadas, N.R.: no reportado por los autores, N.D.: menor al límite de detección

Los POC dominantes en la zona de estudio se destacan en letras negritas 
$100 \%$ de los POC prioritarios, con una concentración importante del isómero delta-HCH como huella molecular del uso de la mezcla técnica del lindano, así como el grupo completo de los "drines" el DDT con el compuesto original en mayor grado, la familia del endosulfán con un dominio del producto de transformación biogeoquímica, el sulfato $(5.4 \mathrm{ng} / \mathrm{g})$ y el heptacloro en su forma primaria. En contraste, Zapata-Pérez et al. (2007) reportaron la presencia de lindano en peces de la especie Ariopsis felis de laguna de Términos, Campeche, con $6.9 \mathrm{ng} / \mathrm{g}$ y un total de $30 \mathrm{ng} / \mathrm{g}$ para la mezcla comercial de este alicíclico $(\mathrm{SHCH})$, mientras que para el pejelagarto (Lepisosteus tropicus) de la misma zona de Términos, Carvalho et al. (2009) detectaron cinco compuestos, entre ellos dieldrín, el grupo del DDT con el dominio de $p, p$ '-DDE $(8.5 \mathrm{ng} / \mathrm{g})$ y sulfato de endosulfán sin registro de lindano (Cuadro VIII). A partir de un trabajo muy completo realizado en la Bahía de Chetumal, Quintana Roo, con el análisis de bagres (Ariopsis assimilis), Noreña-Barroso et al. (2004) reportaron niveles altos de POC muy por encima de los datos existentes para este grupo biológico en los litorales de México; registraron una concentración máxima de $428 \mathrm{ng} / \mathrm{g}$ para $p, p$ 'DDD, lo cual representa un dato probatorio de la antigüedad del compuesto original, pero también encontraron $p, p$ '-DDT en un nivel inferior $(84.9 \mathrm{ng} / \mathrm{g})$, nada despreciable comparativamente con peces de esta familia pertenecientes a otras localidades del GoM. De igual manera, detectaron gran presencia de aldrín (277.6 ng/g), así como de lindano, dieldrín, endrín y heptacloro (Cuadro VIII), lo que se debe muy probablemente a la aplicación continua de este tipo de compuestos clorados con fines de control de vectores para evitar situaciones de paludismo en Chetumal, así como actividades de fumigación constante en los manglares que bordean la región costera del sur de Quintana Roo.

Al igual que para el PacMex, las tortugas marinas del GoM se han incluido en el monitoreo de plaguicidas organoclorados. García-Besné et al. (2015) realizaron un estudio con el análisis de sangre y huevos de la tortuga verde (Chelonia mydas) y de la tortuga carey (Eretmochelys imbricata) en la región de Campeche; pudieron detectar, como era de esperarse, mayor contenido de POC en los huevos de estos reptiles dado el alto contenido lipídico, con el riesgo de transferencia hacia la progenie que esto significa. Comparativamente, el contenido entre especies del grupo del lindano fue mayor en los huevos de tortuga carey, al igual que el de los "drines", sobresaliendo el patrón mostrado por el grupo del DDT en los huevos de la tortuga verde (Ch. mydas), donde el p,p'-DDT fue dominante respecto a sus metabolitos (487.3 vs $38.7 \mathrm{y}$ $89.7 \mathrm{ng} / \mathrm{g})$, mientras que en los de la tortuga carey (E. imbricata) la distribución fue inversa, 2398 ng/g para $p, p$ '-DDD vs. 898.4 y $410.5 \mathrm{ng} / \mathrm{g}$ para $p, p$ '-DDT y $p, p$ '-DDE, respectivamente. Destaca la diferencia en la concentración del DDT entre una especie y otra, ya que el nivel de este insecticida en los huevos de la tortuga carey fue dos veces mayor, situación similar presentada para el heptacloro y su epóxido, lo cual pone de manifiesto que los patrones de exposición son distintos debido principalmente a las zonas de alimentación de cada una de estas especies y las regiones donde habitan gran parte de su vida juvenil. Lo anterior se refleja en los niveles de POC en sangre de estas especies, ya que las concentraciones de la mayoría de los compuestos registrados son de magnitud comparable, con excepción del grupo del heptacloro, cuyo epóxido fue mayor en la sangre de la tortuga carey que en la tortuga verde (19.4 vs. $2.1 \mathrm{ng} / \mathrm{g})$, en tanto que el plaguicida original, el heptacloro, solamente se detectó en los huevos de E. imbricata en una cantidad significativa (Cuadro VIII).

A lo largo de esta revisión se ha podido evidenciar que en ambos litorales de México existe la presencia de compuestos persistentes y de riesgo ambiental (como los plaguicidas organoclorados) en diversas especies de importancia pesquera, ecológica y económica, debido a su gran potencial de bioacumulación resultante de su alta liposolubilidad y biomagnificación. El registro de moléculas primarias como aldrín, dieldrín, endrín, $p, p$ '-DDT, endosulfán, heptacloro y lindano en concentraciones mayores a sus productos de degradación y/o transformación, demuestra un uso reciente de estos agroquímicos pese a la legislación vigente de prohibición para algunos de ellos ("drines") y de restricción para otros (lindano, endosulfán y DDT). Lo anterior, sumado a los residuos antiguos de dichos insumos crea una mezcla compleja de xenobióticos difícil de eliminar una vez que son incorporados a los organismos acuáticos. Estos compuestos tienen, además, características toxicológicas de diversas, son perturbadores hormonales que pueden alterar el éxito reproductivo de las especies y se transmiten a la progenie, como ha demostrado la información sobre acumulación exacerbada de varios compuestos de riesgo en la etapa inicial de vertebrados en peligro de extinción como las tortugas marinas. Cabe destacar que en nuestro país, la legislación sobre plaguicidas organoclorados en ecosistemas naturales es escasa y ambigua, ya que 
como se ha comentado a lo largo de esta revisión, solamente los "drines" están prohibidos desde hace 25 años, mientras que el lindano, el endosulfán y el DDT tienen carácter de restringidos; el resto de los POC, no menos peligrosos, se han dejado de lado. De igual forma, es importante señalar que México cuenta con el Catálogo Oficial de Plaguicidas con una revisión y emisión de 2016, la cual era necesaria y urgente, ya que la anterior databa de 2004. Sin embargo, esta revisión no incluye cambios para los organoclorados; se mantuvo la pequeña lista de compuestos prohibidos y restringidos desde 1991, lo que significa que no se ha avanzado en el cumplimiento de los convenios internacionales que nuestra nación ha firmado y ratificado como el de Estocolmo. Nuestro país tiene la responsabilidad desde 2016 de ejecutar el plan nacional de implementación donde están incluidas las acciones de eliminación definitiva de diversas sustancias persistentes, como los plaguicidas objeto de este trabajo (COFEPRIS 2016, SEMARNAT-ONUDI-IPN 2016).

\section{CONCLUSIONES}

Los organismos costeros y marinos evaluados desde la década de 1970 hasta nuestros días, considerando invertebrados de diversos grupos biológicos como ostiones, almejas, mejillones, camarones y jaibas, así como animales más complejos como peces y reptiles, han registrado la presencia de plaguicidas organoclorados de alto riesgo sistémico, genético y de salud pública, ya que se les considera perturbadores hormonales y carcinógenos. La persistencia de estos compuestos dificulta su eliminación y permite procesos naturales complejos como la biomagnificación, incrementando el riesgo ambiental para las especies costeras y marinas que habitan en los ecosistemas litorales de nuestro país, donde reciben los aportes de agroquímicos usados en prácticas agropecuarias y sanitarias comunes y continuas en diversos estados mexicanos, sin la aplicación de la legislación vigente ni la modificación necesaria en tales actividades humanas en beneficio de los ecosistemas y de la salud humana.

\section{AGRADECIMIENTOS}

Los autores agradecen a la Red Temática de Toxicología de Plaguicidas (CONACyT$262284 / 280045 / 294303$ ) y a los participantes de este estudio.

\section{REFERENCIAS}

APHA-AWWA-WPCF (1992). Métodos normalizados para el analisis de aguas potables y residuales. Ediciones Díaz de Santos, Madrid, España.

Boonyatumanond R., Jaksakul A., Puncharoen P. y Tabucanon M.S. (2002). Monitoring of organochlorine pesticides residues in green mussels (Perna viridis) from the coastal area of Thailand. Environ. Pollut. 119, 245-252. DOI: 10.1016/S0269-7491(01)00329-3

Botello A.V., Rueda-Quintana L., Díaz-González G. y Toledo A. (2000). Persistent organochlorine pesticides (POPs) in coastal lagoons of the Subtropical Mexican Pacific. Bull. Environ. Contam. Tox. 64, 390-397.

DOI: $10.1007 / \mathrm{s} 001280000013$.

Cakirogullari G.C. y Secer S. (2011). Seasonal variation of organochlorine contaminants in bonito (Sarda sarda L. 1758) and anchovy (Engraulis encrasicolus L. 1758) in Black Sea region, Turkey. Chemosphere 85, 1713-1718. DOI:10.1016/j.chemosphere.2011.09.017.

Canada Centre for Inland Waters (1974). Analytical methods manual. Burlington, Ontario.

Carvalho F.P., González-Farías F., Villeneuve J.-P., Cattini C., Hernández-Garza M., Mee L.D. y Fowler S.W. (2002). Distribution, fate and effects of pesticide residues in tropical coastal lagoons of Northwestern Mexico. Environ. Technol. 23(11), 1257-1270.

DOI: $10.1080 / 09593332308618321$.

Carvalho F.P., Villeneuve J.-P., Cattini C., Rendón J. y Mota de Oliveira, J. (2009). Pesticide and PCB residues in the aquatic ecosystems of Laguna de Terminos, a protected area of the coast of Campeche, Mexico. Chemosphere 74, 988-995.

DOI: 10.1016/j.chemosphere.2008.09.092.

COFEPRIS (2016). Catálogo Oficial de Plaguicidas. Comisión Federal para la Protección contra Riesgos Sanitarios, Ciudad de México, 50 pp.

DOF (1991). Relación de plaguicidas prohibidos para su importación, fabricación, formulación, comercialización y uso en México. Comisión Intersecretarial para el Control del Proceso y uso de Plaguicidas, Fertilizantes y Sustancias Tóxicas. Diario Oficial de la Federación, 3 de enero.

Galindo-Reyes J.G., Fossato V.U., Villagrana-Lizarraga C. y Dolci F. (1999). Pesticides in water, sediments, and shrimp from a coastal lagoon off the Gulf of California. Mar. Pollut. Bull. 38(9), 837-841. DOI: 10.1016/S0025-326X(99)00086-7

García-Besné G., Valdespino C. y Rendón-von Osten J. (2015). Comparison of organochlorine pesticides and PCB residues among hawksbill (Eretmochelys imbricata) and green (Chelonia mydas) turtles in the Yucatan 
Peninsula and their maternal transfer. Mar. Pollut. Bull. 91, 139-148. DOI: 10.1016/j.marpolbul.2014.12.015. García-Solorio L., Noreña-Barroso E. y Capella-Vizcaíno, S. (2014). Plaguicidas organoclorados en huevos de la tortuga golfina Lepidochelys olivacea (Eschscholtz, 1829), en las costas del estado de Sinaloa, México. En: Pacífico mexicano. Contaminación e impacto ambiental: diagnóstico y tendencias (Botello A.V., Páez-Osuna F., Méndez-Rodríguez L., BentacourtLozano M., Álvarez-Borrego S. y Lara-Lara R., Eds.). UAC, UNAM-ICML, CIAD-MAZATLÁN, CIBNOR, CICESE, México, pp. 43-56.

Gardner S.C., Pier M. D., Wesselman R. y Juárez J.A. (2003). Organochlorine contaminants in sea turtles from the Eastern Pacific. Mar. Pollut. Bull. 46, 10821089. DOI: $10.1016 / \mathrm{S} 0025-326 \mathrm{X}(03) 00254-6$

Gold-Bouchot G., Silva-Herrera T. y Zapata-Pérez O. (1993). Chlorinated pesticides in the rio Palizada, Campeche, Mexico. Mar. Pollut. Bull. 26(11), 648-650. DOI: 10.1016/0025-326X(93)90505-E

Granados-Galván I.A., Rodríguez-Meza D.G., LunaGonzález A. y González-Ocampo H.A. (2015). Human health risk assessment of pesticide residues in snappers (Lutjanus) fish from the Navachiste Lagoon complex, Mexico. Mar. Pollut. Bull. 97, 178-187.

DOI: 10.1016/j.marpolbul.2015.06.018

Holden A. V. y Marsden K. (1969). Single-stage clean-up of animal tissue extracts for organochlorine analysis. J. Chromatogr. 44, 481-492. DOI: $10.1016 / \mathrm{S} 0021-9673(01) 92572-5$

IARC (2011). Monographs on the evaluation of carcinogenic risks to humans. Agents classified by the IARC. Monographs, volumes 1-102. List of classification. International Agency for Research on Cancer.

Labrada-Martagón V., Tenorio-Rodríguez P.A., MéndezRodríguez L.C. y Zenteno-Savín T. (2011). Oxidative stress indicators and chemical contaminants in East Pacific green turtles (Chelonia mydas) inhabiting two foraging coastal lagoons in the Baja California peninsula. Comp. Biochem. Phys. Part C 154, 65-75. DOI: 10.1016/j.cbpc.2011.02.006

Noreña-Barroso E., Simá-Álvarez R., Gold-Bouchot G. y Zapata-Pérez O. (2004). Persistent organic pollutants and histological lesions in Mayan catfish Ariopsis assimilis from the Bay of Chetumal, Mexico. Mar. Pollut. Bull. 48, 263-269.

DOI: 10.1016/j.marpolbul.2003.08.001.

Osuna-Flores I. y Riva M.C. (2002). Organochlorine pesticide residue concentrations in shrimps, sediments, and surface water from Bay of Ohuira, Topolobampo, Sinaloa, Mexico. Bull. Environ. Contam. Tox. 68, 532-539.

DOI: $10.1007 / \mathrm{s} 00128-001-0287-4$.
Osuna-López I., Frías-Espericueta M.G., López-López G., Izaguirre-Fierro G., Zazueta-Padilla H., Aguilar-Juárez M., Correa-González E.M., Bautista-Covarrubias J.C., Cervantes-Atondo J.A., Sánchez-Osuna L. y Voltolina D. (2014). Niveles de concentración de pesticidas organoclorados en moluscos bivalvos del noroeste de México. En: Pacífico mexicano. Contaminación e impacto ambiental: diagnóstico y tendencias (Botello A.V., Páez-Osuna F., Méndez-Rodríguez L., Bentacourt-Lozano M., Álvarez-Borrego S. y Lara-Lara R., Eds.). UAC, UNAM-ICML, CIADMAZATLÁN, CIBNOR, CICESE, pp. 33-42.

Páez-Osuna F., Osuna-López J.I. y Vázquez-Botello A.V. (1998). Biomonitoreo de la contaminación en las aguas costeras del Pacífico subtropical Mexicano: metales pesados, plaguicidas e hidrocarburos del petróleo. Informe técnico académico final. Proyecto CONACyT 0185PT, México.

Palmerín R.C., Ponce-Vélez G. y Botello A.V. (2014). Evaluación de plaguicidas organoclorados en sedimentos y organismos filtradores de la laguna de Alvarado, Veracruz, México. En: Golfo de México. Contaminación e impacto ambiental: diagnóstico y tendencias (Botello A.V., Rendón von-Osten J., Benítez J.A. y Gold-Bouchot G., Eds.). UAC, UNAM-ICML, CINVESTAV-Unidad Mérida, p. 285-308.

SEMARNAT-ONUDI-IPN (2016). Plan Nacional de Implementación del Convenio de Estocolmo en México. Secretaría de Medio Ambiente y Recursos Naturales, Organización de las Naciones Unidas para el Desarrollo Industrial, Centro Mexicano de Producción más Limpia/Instituto Politécnico Nacional, Ciudad de México, $136 \mathrm{pp}$.

Ponce-Vélez G. (2012). Los contaminantes orgánicos persistentes en la laguna El Yucateco, Tabasco, México: Una década de estudio. Tesis de Doctorado. Posgrado en Ciencias de la Tierra, Universidad Nacional Autónoma de México. México, D.F. México. 284 pp.

Rendón von Osten J. (2014). Plaguicidas. En: Golfo de México. Contaminación e Impacto Ambiental: Diagnóstico y Tendencias. (A. V. Botello, J. Rendón von-Osten, J. A. Benítez y G. Gold-Bouchot, Eds. UAC, UNAM-ICML, CINVESTAV-Unidad Mérida, p. 181-182.

Robledo-Marenco L., Botello A.V., Romero-Bañuelos C.R. y Díaz-González G. (2006). Presence of persistent organochlorine pesticides in estuaries of the subtropical Mexican Pacific. Int. J. Environ. Pollut. 26(1/2/3), 284-294. DOI: 10.1504/IJEP.2006.009112.

Rosales M.T.L, Botello A.V., Bravo H. y Mandelli E.F. (1979). PCBs and organochlorine insecticides in oysters from coastal lagoons of the Gulf of Mexico, Mexico. Bull. Environ. Contam. Tox. 21, 652-656. 
Rosales M.T.L. y Escalona R.L. (1983). Organochlorine residues in organisms of two different lagoons of Northwest Mexico. Bull. Environ. Contam. Tox. 30, 456-463.

Rueda Q.L., Botello A.V. y Díaz G. (1997). Presencia de plaguicidas organoclorados en dos sistemas lagunares del estado de Chiapas, México. Rev. Int. Contam. Ambie. 13(2), 55-61.

UNEP (1982). Determination of DDT's and PCB's in selected marine organisms. Reference methods for marine pollution studies No. 14. United Nations Environment Programme, Genova.

UNEP (1988). Determination of DDT's and PCB's by capillary gas chromatography and electron capture detection. Reference methods for marine pollution studies No. 40. United Nations Environmental Programme, Genova.

UNEP (1991). Sampling of selected marine organisms and sample preparation for the analysis of chlorinated hidrocarbons. Marine Pollution Studies No. 12, Rev. 2.

UNEP/FAO/IOC/IAEA (1986). Determination of DDT's, PCB's in selected marine organisms by packed column gas chromatography. Reference methods for marine pollution studies No. 14. United Nations Environment Programme.

UNEP/FAO/IOC/IAEA (1991). Sampling of selected marine organisms and sample preparation for the analysis of chlorinated hydrocarbons. Reference methods for marine pollution studies No. 12, Rev. 2. United Nations Environment Programme. Nairobi, Kenya.

UNEP/FAO/IOC/IAEA(1993). Guidelines for monitoring chemical contaminants in the sea using marine organisms. Reference methods for marine pollution studies No. 16. United Nations Environment Programme, Nairobi, Kenya.

UNEP/IOC/IAEA (1988). Determination of DDT's and PCBs in selected marine organisms by capillary column gas chromatography. Reference methods for marine pollution studies No. 40. United Nations Environment Programme, Nairobi, Kenya.

Zapata-Pérez O., Ceja-Moreno V., Roca-Olmos M., Pérez M.T., del Río-García M., Yarto M., Mendoza-Cantú A., Ize-Lema A.I., Gavilán-García A., Sánchez-Teyer L.F. y Gold-Bouchot G. (2007). Ecotoxicological effects of POPs on ariidae Ariopsis felis (Linnaeus, 1766) from three coastal ecosystems in the Southern Gulf of Mexico and Yucatan Peninsula. J. Environ. Sci. Health A 42(10), 1513-1520. DOI: $10.1080 / 10934520701480961$

Zhang H., Wang Z., Lu B., Zhu C., Wu G. y Walter V. (2007). Occurrence of organochlorine pollutants in the eggs and dropping-amended soil of Antarctic large animals and its ecological significance. Sci. China Ser. D. 50, 1086-1096. DOI: 10.1007/s11430-007-0021-0. 